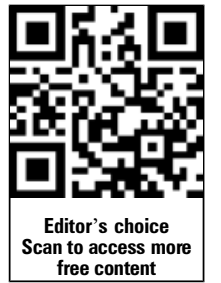

${ }^{1}$ Department of Neurology, Chiba University School of Medicine, Chiba, Japan

${ }^{2}$ Autonomic and Neurovascular Medicine Unit, Division of Brain Sciences, Faculty of Medicine, Imperial College London at St Mary's Hospital, London, UK

${ }^{3}$ Autonomic Unit, National Hospital for Neurology and Neurosurgery, Queen Square/ Division of Clinical Neurology, Institute of Neurology, University College London, London, UK

\section{Correspondence to} Dr M Asahina, Department of Neurology, Chiba University

School of Medicine, 1-3-1 Inohana, Chuo-ku, Chiba 260-8670, Japan;

asahina@faculty.chiba-u.jp

Received 18 July 2012 Accepted 10 August 2012 Published Online First 1 September 2012

\footnotetext{
To cite: Asahina $M$, Vichayanrat E, Low DA et al. I Neurol Neurosurg Psychiatry 2013;84: 674-680.
}

\title{
Autonomic dysfunction in parkinsonian disorders: assessment and pathophysiology
}

\author{
Masato Asahina, ${ }^{1}$ Ekawat Vichayanrat, ${ }^{2,3}$ David A Low, ${ }^{2,3}$ Valeria lodice, ${ }^{2,3}$ \\ Christopher J Mathias ${ }^{2,3}$
}

\section{ABSTRACT}

Parkinson's disease (PD) is a progressive neurodegenerative disorder characterised by motor dysfunction (parkinsonism) and several non-motor features. Dysautonomia is a significant non-motor feature as well as a neuropsychiatric symptom. Autonomic dysfunction can occur even in the early stages of PD, often preceding the onset of the classic motor symptoms of PD. The patterns of autonomic features in PD are different from other parkinsonian disorders. Detection of autonomic dysfunction may therefore be helpful in diagnosing PD in the early or pre-motor stages, and/or in differentiating it from other parkinsonian disorders, such as multiple system atrophy and progressive supuranuclear palsy. The aim of this review is to describe aspects of autonomic dysfunction, including symptoms, assessment and pathophysiology, resulting from autonomic impairment in PD and other parkinsonian syndromes.

\section{INTRODUCTION}

The autonomic nervous system innervates and influences every organ in the body. This complex system is mediated by two major efferent pathways, the sympathetic and parasympathetic nervous systems, which obtain afferent signals from different parts of the body, neurons in the spinal cord and cerebral autonomic centres primarily in the hypothalamus, midbrain and brainstem. The gastrointestinal tract is controlled by the sympathetic and parasympathetic nervous systems and, additionally, the enteric nervous system which, through Meissner's and Auerbach's plexuses, control motility and secretion in the small and large intestines.

Non-motor features of Parkinson's disease (PD) are increasingly being defined and include dysfunction of the autonomic nervous system. Autonomic dysfunction was previously reported in the advanced stage of PD but recent evidence suggests it may occur even in the early stage of PD, independent of medication. Autonomic features, that include constipation, often precede the onset of motor symptoms. ${ }^{1}$ This is consistent with the Braak hypothesis suggesting that PD patients have Lewy body pathology in autonomic centres and nerves that include the dorsal motor nucleus of the glossopharyngeal and vagal nerves, gastrointestinal submucosal plexus and postganglionic sympathetic nervous system, which is present in the pre-motor stage before nigral involvement. ${ }^{2}$ These lesions may result in autonomic dysfunction. Therefore, detection of autonomic dysfunction may be helpful to diagnose PD in the early motor or premotor stages, or to differentiate between PD and other parkinsonian disorders, such as multiple system atrophy (MSA) and progressive supuranuclear palsy (PSP). The aim of this review is to describe aspects of autonomic dysfunction, including symptoms, assessment and pathophysiology, resulting from autonomic impairment in PD and other parkinsonian syndromes.

\section{AUTONOMIC SYMPTOMS AND SIGNS \\ Cardiovascular symptoms and signs \\ Postural symptoms (orthostatic hypotension)}

Orthostatic hypotension may cause considerable problems at various stages of PD and particularly in the advanced stages. It is defined as a fall in systolic blood pressure of $\geq 20 \mathrm{~mm} \mathrm{Hg}$ or in diastolic blood pressure $\geq 10 \mathrm{~mm} \mathrm{Hg}$ on either standing or head-up tilt to at least $60^{\circ}$ degree within $3 \mathrm{~min}^{3} \mathrm{~A}$ drop of systolic blood pressure of $\geq 30 \mathrm{~mm} \mathrm{Hg}$ has recently been suggested in patients with recumbent hypertension. ${ }^{4}$ Cerebral perfusion is maintained by autoregulation of cerebral blood flow if systemic blood pressure is within a certain range (mean arterial pressure $60-200 \mathrm{~mm} \mathrm{Hg}$ ). Patients may not complain of postural symptoms due to cerebral hypoperfusion despite having orthostatic hypotension when their mean arterial pressure is above $60 \mathrm{~mm} \mathrm{Hg}$.

Symptoms related to orthostatic hypotension are mainly due to reduction of blood flow to various organs, especially the brain. Cerebral hypoperfusion can result in posturally induced dizziness, visual disturbances (blurring, colour change, whiteout, grey-out, etc), transient cognitive impairment and loss of consciousness (syncope). Hypoperfusion of muscles may result in headache, neck pain ('coat hanger' ache) and lower back pain. Orthostatic hypotension may cause fatigue, chest pain, dyspnoea and fall. Some may have relatively few symptoms except occasionally, suggesting that they have somehow adapted to a lower blood pressure when upright until it is lowered by additional factors causing vasodilatation, such as food ingestion, exercise, heat or drugs with hypotensive properties, including some antiparkinsonian agents. The prevalence of these postural hypotensive symptoms in PD has been reported in 56\% of PD patients and in $16 \%$ of age matched healthy controls, with syncope in $4 \%$ of PD and $1 \%$ of controls. ${ }^{5}$ The incidence of these symptoms increases with progression of the disease. ${ }^{6}$ 


\section{Supine (recumbent) hypertension}

PD patients with orthostatic hypotension may also have supine hypertension, defined as a systolic blood pressure $\geq 150 \mathrm{~mm} \mathrm{Hg}$ or diastolic blood pressure $\geq 90 \mathrm{~mm} \mathrm{Hg.}{ }^{7}$ This may be a problem, especially at night if they lie supine, with a reversal of the circadian change in blood pressure. Supine hypertension is usually asymptomatic, but some patients report headfullness or a throbbing headache while lying flat. It may contribute to ventricular hypertrophy, ${ }^{8}$ renal dysfunction ${ }^{9}$ and intracerebral haemorrhage. The mechanisms of supine hypertension include impaired baroreflex activity, inappropriate sympathetic tone (eg, a higher residual sympathetic activity) while supine, ${ }^{10}$ altered endocrine systems and drugs used to reverse orthostatic hypotension.

\section{Postprandial hypotension}

In normal healthy individuals, food ingestion does not change systemic blood pressure, as changes in gastrointestinal and pancreatic hormones are accompanied by compensatory cardiac and regional haemodynamic responses. Previous studies in patients with autonomic failure indicate that postprandial hypotension occurs even while supine, because of impaired compensatory mechanisms for postprandial splanchnic blood pooling. Carbohydrates are more likely to lower blood pressure postprandially than protein or fat. Postprandial hypotension is known to aggravate orthostatic hypotension, ${ }^{11}$ and may be more prominent in the morning and after large meals. In PD, there are varying reports on the extent of postprandial hypotension. In untreated PD, a small postprandial fall in blood pressure while supine has been reported. ${ }^{12}$ Others have noted a greater incidence and degree of postprandial hypotension in elderly parkinsonian patients that was more frequent than orthostatic hypotension. ${ }^{13}$

\section{Exercise induced and post exercise hypotension}

In patients with autonomic failure, exercise, even when performed supine, induces a fall in blood pressure, and worsens orthostatic hypotension. ${ }^{14}$ Exercise induced hypotension persists after exercise and accentuates post exercise orthostatic hypotension due to the continuing uncompensated vasodilation in previously active skeletal muscles. In patients with exercise induced hypotension, hypotensive symptoms such as dizziness, blurring and syncope often occur soon after cessation of exercise-for example, just after walking up stairs-presumably because of lack of the calf muscle pump which helps support the circulation during walking.

\section{Gastrointestinal symptoms}

Upper gastrointestinal symptoms

Sialorrhoea is a common symptom in PD. ${ }^{5}$ Previous studies exclude increased secretion of saliva in PD and indicate that saliva production is decreased, indicating that sialorrhoea may result from reduced and inefficient swallowing in $\mathrm{PD}^{15}$ Sialorrhoea is thus likely to be a motor rather than an autonomic symptom in PD. PD patients may present with common upper gastrointestinal symptoms, such as nausea, early satiety, loss of appetite and abdominal distension, considered to be due to gastrointestinal dysmotility. Vomiting is not rare in PD. ${ }^{5}$

\section{Constipation}

Constipation is variably defined as bowel movements less than three times a week, regular laxative use and/or difficulty during defecation. It is the most frequent lower bowel symptom and can be a major problem in PD. Approximately half of PD patients have constipation, with increasing severity in the later stages. ${ }^{5}$ It may not be useful as a predictor of PD, because constipation is common in healthy subjects, particularly women. However, in young men, constipation may be considered a significant risk factor for developing PD. Epidemiological studies have also shown that men who have bowel movements less than once daily compared with two times or more daily have a four times higher risk of PD. ${ }^{1}$ Intestinal abnormalities, including megacolon, as a result of intestinal pseudo obstruction, have also been reported in PD.

\section{Urinary problems}

Urinary dysfunction is common in PD with a prevalence of more than $50 \%$ using validated questionnaires. ${ }^{5}{ }^{6}$ The exact figure attributed to PD is difficult to confirm because benign prostate hyperplasia and idiopathic detrusor overactivity often occur in otherwise normal individuals with increasing age. In the majority of PD patients, bladder symptoms occur after the development of motor symptoms. ${ }^{16}$ Urinary features are categorised into storage symptoms and voiding symptoms. Storage symptoms, such as urinary frequency, nocturnal urine (sleep is disturbed at least once a night), urgency and incontinence, are more common, while severe voiding symptoms (delays in initiating urination, poor or prolongation of urine stream) are not particularly common in early PD. An excess of $100 \mathrm{ml}$ of residual urine or enuresis is also rare in PD. ${ }^{17}$

\section{Sexual dysfunction}

Erectile dysfunction is frequently reported in PD compared with age matched healthy controls. ${ }^{5}$ In a recent epidemiological study, erectile dysfunction was associated with a 2.7-4 times higher risk of developing PD compared with those without this problem at the same age. ${ }^{18}$ Sexual dysfunction in PD might be caused by multiple factors, including the disease itself depleting dopamine or associated features, such as depression and anxiety.

\section{Abnormal sweating and heat intolerance}

Sweating abnormalities occur in $30-55 \%$ of PD patients. ${ }^{5} 6$ Hyperhidrosis or hypo/anhidrosis may occur. Hyperhidrosis is usually regional and on the upper body, such as the head, face, neck and chest, and often occurs during the off period associated with the wearing off phenomenon. Some have hyperhidrosis during the on period, often with dyskinesia. ${ }^{19}$ Hypohidrosis may occur more frequently in the lower body.

PD patients may have heat intolerance. ${ }^{5}$ The parkinsonismhyperpyrexia syndrome, also known as neuroleptic malignant syndrome, is a life threatening complication of PD. It is clinically characterised by hyperthermia, aggravated parkinsonism, including increased muscle rigidity, depressed consciousness, autonomic dysfunction and elevated serum creatine kinase levels. It may be induced by interruption or reduction of antiparkinsonian drug treatment, infection and hot weather. ${ }^{20}$

\section{Pupillary abnormalities}

There are only a few reports on pupil abnormalities in PD indicating slow pupillary responses to light and pain, ${ }^{21}$ and the usefulness in PD remains unclear. Clinical examination usually does not reveal pupillary abnormalities in PD.

\section{AUTONOMIC TESTS}

As autonomic symptoms are diverse, a range of autonomic investigations need to be used for evaluating the site and extent of an autonomic lesion. Autonomic investigation is of value in diagnosing PD and differentiating PD from other parkinsonian disorders, such as MSA. 


\section{Cardiovascular system}

Head-up tilting

Head-up tilting is a conventional and useful cardiovascular autonomic function test, particularly for detecting orthostatic hypotension. In the clinic, this can also be performed by lying down and then sitting or standing. Blood pressure is preferably measured continuously during the test, but regular upper arm sphygmomanometry measurements can suffice. The prevalence of orthostatic hypotension in PD previously reported in the literature has varied but a recent meta-analysis of 25 studies calculated a prevalence of $30 \%$ with large statistical heterogeneity between studies, ${ }^{22}$ likely due to the type of study, participants and methodologies employed. Furthermore, some patients do not have symptoms even though they have orthostatic hypotension, and as a result it is not recognised by clinicians. It is now clear that orthostatic hypotension can be seen in any stage of $\mathrm{PD}$ but it is more prevalent in later stages. ${ }^{22}$

\section{Valsalva manoeuvre and pressor stimuli tests}

The blood pressure response to the Valsalva manoeuvre, during which intrathoracic pressure is raised to a maximum of $40 \mathrm{~mm}$ $\mathrm{Hg}$, depends on the integrity of the baroreflex pathways and is frequently abnormal in patients with autonomic failure and as such is frequently used to evaluate cardiovascular autonomic function. Abnormal baroreflex-cardiovagal gain responses, calculated during the Valsalva manoeuvre, are evident in PD patients with orthostatic hypotension and in up to $25 \%$ of PD patients. ${ }^{23}$

\section{Ambulatory $24 \mathrm{~h}$ blood pressure recording}

Ambulatory $24 \mathrm{~h}$ blood pressure recording is useful to detect supine (nocturnal) hypertension, as well as postprandial or orthostatic hypotension, which may be strong contributors to abnormal circadian blood pressure rhythms. Postprandial hypotension is most prevalent after the first two meals of the day. ${ }^{24}$ Lack of nocturnal blood pressure fall (non-dipping) due to reversal of the normally expected circadian fall is common in PD with autonomic failure. ${ }^{25}$ In addition, $24 \mathrm{~h}$ blood pressure recordings provide clinicians with useful information on daily fluctuations in blood pressure, which is helpful for developing a treatment strategy (administration time and choice of medication) for postprandial hypotension, orthostatic hypotension and nocturnal supine hypertension.

\section{Meal challenge test}

The meal challenge test helps detect postprandial hypotension. Usually, blood pressure is measured in a supine posture in the fasting state and up to $120 \mathrm{~min}$ after a standard test meal. Postprandial hypotension is defined analogously to orthostatic hypotension as a decrease in systolic blood pressure of $20 \mathrm{~mm} \mathrm{Hg} .{ }^{11}$

\section{Exercise testing}

In PD patients evaluated both on and off medication, elevations in blood pressure and heart rate during exercise are lower compared with normal controls and appear to be disease related and not affected by medication used to treat PD. ${ }^{26}$ Exercise testing can identify exercise induced and/or post exercise hypotension in patients with suspected autonomic failure. ${ }^{14}$

\section{Plasma norepinephrine}

Plasma norepinephrine levels reflect sympathetic postganglionic activity, although with limitations as only $10 \%$ of norepinephrine released into the synaptic cleft enters the plasma, and its clearance may be reduced in patients with postganglionic sympathetic failure. ${ }^{27}$ Patients with predominant sympathetic postganglionic lesions, such as pure autonomic failure, have reduced plasma norepinephrine levels. The norepinephrine response to head-up tilting or standing is diminished due to disturbed baroreflex function in patients with autonomic failure. ${ }^{27} \mathrm{PD}$ patients without orthostatic hypotension have been reported to have no major changes in baseline supine resting and orthostatic plasma norepinephrine levels. In the early stage of PD without orthostatic hypotension, plasma norepinephrine may rise slightly in some patients. ${ }^{28}$ In contrast, PD with orthostatic hypotension may show subnormal baseline levels as well as subnormal orthostatic increments, consistent with autonomic failure. ${ }^{28}$

\section{Heart rate variability}

Heart rate variability, analysed by time domain or frequency domain methods, is a non-invasive method used to assess autonomic function by evaluating the modulatory effects of neural mechanisms on the sinus node. Time domain analyses of heart rate variability might be normal but may decrease during mid and late stages of $\mathrm{PD}^{29}$ Spectral (frequency domain) analysis may provide indices of sympathetic and parasympathetic function, which have been shown to be abnormal in PD patients, particularly in indexes of sympathetic function, although these measures have recently been proposed to reflect baroreflex, rather than sympathetic nerve activity function. ${ }^{30}$

\section{Skin vasomotor reflex}

Some procedures, such as deep inspiration, mental stress and isometric exercise, provoke reduced cutaneous blood flow in the palm or sole, and this response is termed the skin vasomotor reflex. An attenuated skin vasomotor reflex was present in patients with Lewy body disease. ${ }^{31}$ These observations are consistent with Lewy body pathology observed in the raphe nucleus which plays an important role in the skin vasomotor reflex ${ }^{32}$ and may also reflect postganglionic sympathetic involvement seen in patients with Lewy body disorders.

\section{Myocardial scintigraphy with ${ }^{123}$ I-meta-iodobenzylguanadine}

Imaging techniques can determine the integrity of cardiac sympathetic innervation, using myocardial scintigraphy of the physiological analogue of norepinephrine, ${ }^{123}$ I-meta-iodobenzylguanadine (MIBG), as well as ${ }^{18} \mathrm{~F}$-fluorodopamine, and positron emission tomography scanning. ${ }^{33}$ In PD there is impaired uptake, suggesting cardiac sympathetic denervation. ${ }^{34}$ This can occur in the absence of clinical features suggesting autonomic failure and at an early stage of PD, and also diminishes as the disease progresses. ${ }^{35}$ Studies with similar techniques indicate that PD patients with autonomic failure have decreased noradrenergic innervation in the renal cortex ${ }^{36}$ and thyroid, ${ }^{37}$ which may possibly contribute to dysregulation of circulatory homeostasis in PD. ${ }^{36}$ Previous studies using MIBG to identify patients with PD from normal subjects showed a sensitivity of $88 \%$ and a specificity of $85 \%{ }^{34}$ It is unclear whether the severity of PD and autonomic dysfunction are correlated with MIBG uptake.

\section{Gastrointestinal system}

Swallowing and oesophageal motility

A previous study showed that $63 \%$ of PD patients have abnormalities in the modified barium swallow test. ${ }^{38}$ Dysphagia occurs in both oral and pharyngeal phases in PD patients. ${ }^{39}$ Although oropharyngeal dysphasia is common among PD patients, unlike other parkinsonian disorders such as MSA, it is usually asymptomatic in the early stages and features in the 
advance stages. Oesophageal dysphagia is also prevalent in PD, as demonstrated by abnormalities noted during videofluoroscopy ${ }^{40}$ and manometry. ${ }^{41}$

\section{Gastric emptying studies}

Various methodologies have been used to evaluate gastric emptying. A solid or liquid meal is mixed with a small amount of radioactive material, and the amount of radioactivity in the stomach is measured for several hours. The ${ }^{13} \mathrm{C}$ octanoic acid or ${ }^{13} \mathrm{C}$-acetate breath test is also used to measure gastric emptying of solids. In PD, disturbed gastric emptying has been found in the early untreated stage. ${ }^{42}$

\section{Electrogastrogram}

Electrogastrography (EGG) using surface electrodes can record gastric slow waves (gastric pacemaker rhythm) at $\sim 3$ cycles $/ \mathrm{min}$, which originate from the pacemaker cells on the major curvature of the stomach. EGG can be clinically used to evaluate gastric motility. ${ }^{43}$ Slow waves have been reported to be irregular in PD patients. ${ }^{44}$

\section{Colon transit time and anorectal manometry}

Recent studies suggest that delayed gastric emptying results from delayed bowel transport with or without abnormal anorectal evacuation. Colon transit time was longer in PD patients ${ }^{45}$ and thought to be due to degeneration of parasympathetic nuclei and myenteric neurons. ${ }^{46}$ Anorectal manometric abnormalities, including lower basal sphincter pressure and abnormal phasic contractions during voluntary contraction, have also been observed. ${ }^{47}$ Pelvic floor dyssynergia and the lack of puborectalis relaxation might contribute to constipation in PD. ${ }^{48}$

\section{Urinary function tests: urodynamic study and sphincter electromyography}

Cystometric analysis of the storage phase indicates reduced bladder capacity with detrusor overactivity in PD as well as uninhibited external sphincter relaxation. ${ }^{49}$ These findings may be major contributing factors to an overactive bladder in PD.

The pressure-flow analysis of the voiding phase in PD has shown a weak detrusor in $40 \%$ of male and $66 \%$ of female patients. ${ }^{49}$ A subset of PD patients had detrusor overactivity during the storage phase but weak detrusor on voiding. This condition, known as detrusor hyperactivity with impaired contractile function, has recently been estimated to occur in $18 \%$ of PD patients. ${ }^{50}$

Detrusor-external sphincter dyssynergia or pseudodyssynergia may be detected in $\mathrm{PD},{ }^{51}$ but the prevalence is considered to be low. In contrast, a pressure-flow analysis revealed that half of PD patients show mild urethral obstruction. Sphincter electromyography indicates that neurogenic changes in sphincter motor unit potentials are not common in PD. ${ }^{49}$

\section{Sudomotor system}

Thermoregulatory sweating test

In thermoregulatory sweat testing, the patterns of anhidrosis in PD may vary, such as the truncal and proximal limb regions (central pattern), segmental regions and myelopathic distributions. ${ }^{52}$ This wide spectrum of anhidrotic patterns implies that the initial involvement and progression of autonomic cellular loss varies considerably.

\section{Quantitative Sudomotor Axon Reflex Test}

The Quantitative Sudomotor Axon Reflex Test is used for assessing postganglionic sudomotor function and is often used in conjunction with thermoregulatory sweating testing. There have been only a few studies using this method, indicating reduced ${ }^{53}$ and normal axon reflex sweating. ${ }^{54}$

Sympathetic skin response and sympathetic sweat response Sympathetic skin responses (SSR) have been used for evaluating sudomotor function by measuring evoked electrodermal activity, which reflects sweat gland activity, on the hand or foot. SSR mainly reflects emotional sweating on the palm or sole. However, the SSR is less reliable as a quantitative index of sweating. In contrast, the sympathetic sweat response, where the amount of sweat output is measured by a sudorometer or capacitance hygrometry, is a useful quantitative method for evaluating sudomotor function. Responses may be reduced or absent in PD patients. $^{31}$

\section{Skin biopsy}

In skin biopsies from the lower thigh, autonomic innervation of the blood vessels, sweat glands and erector pili muscles have been reported to be reduced in PD. ${ }^{55}$ In autopsy cases of PD, $\alpha$-synuclein pathology was demonstrated in the unmyelinated fibres of the abdominal dermis in $20(23.5 \%)$ of 85 patients who also had Lewy body pathology in the brain. ${ }^{56}$ However, in studies with skin biopsy, abnormal $\alpha$-synuclein accumulation in unmyelinated fibres was present in the dermis of the thoracic wall but not in the lower limb in two (10\%) of 20 PD patients. ${ }^{57}$ The same group also retrospectively examined abdominal skin in 142 patients with Lewy body pathology in the CNS and demonstrated that the sensitivity of Lewy body pathology in the skin was $70 \%$ in PD. The discrepancy between results of autopsy based and skin biopsy studies may be explained by the differences in the sites for tissue samples, the size of skin tissue examined and the number of examined sections.

\section{PATHOPHYSIOLOGY OF AUTONOMIC DYSFUNCTION} Involvement of the autonomic nervous system in PD $\mathrm{PD}$ is characterised by deposition of abnormally phosphorylated $\alpha$-synuclein. The aggregates are typically found in neurons as Lewy bodies. ${ }^{2}$ Alongside the hallmark degeneration of the substantia nigra in PD, Lewy bodies and cell loss are detected in: (1) autonomic regulatory areas such as the hypothalamus, parabrachial nucleus, intermediate reticular zone of the medulla, locus coeruleus and raphe; (2) preganglionic parasympathetic regions, such as the Edinger-Westphal nucleus and dorsal vagal motor nuclei; (3) preganglionic sympathetic neurons in the intermediolateral cell column and (4) neurons in paravertebral and paravertebral autonomic ganglia. ${ }^{58}$ Histological loss of neurons and Lewy body accumulation in sympathetic ganglia, ${ }^{59}$ and cardiac sympathetic denervation in several studies are also evident. ${ }^{60}$ In addition, $\alpha$-synuclein pathology has been reported to be shown in the ventrolateral medulla (pressor centre) ${ }^{61}$ and the enteric plexus in PD patients. ${ }^{46}$ The lesions in autonomic regulatory areas could be key causes of cardiovascular, sudomotor, bladder and bowel dysfunction. Evidence suggests that urinary dysfunction is also a process of degeneration in the substantia nigra as urinary dysfunction in PD appears to correlate with PD severity. ${ }^{62}$

In the Braak staging model of $\mathrm{PD},{ }^{2}$ the dorsal motor nucleus of the vagal nerves and anterior olfactory are proposed to be affected in stage 1 , and the locus coeruleus, raphe and lateral tegmental nucleus in stage 2 . In stage 3 , the substantia nigra is affected and it is in this stage when the hallmark motor symptoms of PD develop in accordance with large dopaminergic 
neuron loss. ${ }^{2}$ In addition, Braak et al has reported that $\alpha$-synuclein immunoreactive inclusions were found in neurons of the enteric plexus in individuals with incidental Lewy body disease. ${ }^{46}$ It was proposed that PD could start from impairments in the gastrointestinal tract, earlier than the anterior olfactory nuclei, dorsal motor nucleus of the vagal nerve and substantia nigra. Sympathetic postganglionic neurons may be involved in the early or premotor stage. ${ }^{60}$ Impaired cardiovascular autonomic control and gastrointestinal disturbances may predate motor symptoms in PD, but this has not been confirmed clinically.

\section{Effect of antiparkinsonian treatment on the autonomic nervous system}

Antiparkinsonian medication may cause autonomic dysfunction. In the periphery, a considerable amount of orally administrated L-dopa is transformed into dopamine, which is an important regulator of systemic blood pressure through vasodilation and decreased catecholamine release, ${ }^{63}$ and dopaminergic receptors are widely distributed throughout both the central and peripheral autonomic nervous systems. Therefore, L-dopa may affect cardiovascular function. However, the effect of oral levodopa, with or without a peripheral decarboxylase inhibitor (DCI), on blood pressure and orthostatic hypotension is controversial, although DCIs have been reported to have no effect on blood pressure. ${ }^{64}$ In healthy individuals, intravenous levodopa infusion has been reported to lower blood pressure ${ }^{65}$ whereas oral L-dopa with DCI does not. ${ }^{66}$ With regards to PD patients, in several previous studies, acute or chronic administration of oral L-dopa has not been shown to affect cardiovascular reflexes, ${ }^{67} 68$ whereas some have shown that L-dopa produces mild orthostatic hypotension ${ }^{69}$ and lowers resting blood pressure. ${ }^{69} 70$ In contrast, one report has shown that orthostatic hypotension improved after 6 months of oral L-dopa therapy. ${ }^{70}$

Dopamine agonists could lower blood pressure and could cause orthostatic hypotension. ${ }^{63}$ Monoamine oxidase inhibitors, such as selegiline, have also been shown to diminish cardiovascular autonomic responses in PD patients and to increase the risk of orthostatic hypotension. ${ }^{70}$ The effect of catechol-Omethyl-transferase inhibitor therapy on the cardiovascular system is not significant in PD patients. ${ }^{71}$

Dopamine also affects gastrointestinal and colonic motility; it reduces gastric migrating motor complex and gastric motility ${ }^{72}$ whereas it may increase colonic motor activity. ${ }^{73}$ Therefore, it is possible that L-dopa affects bowel function in PD. However, no reports are available that have examined whether L-dopa might change gut function in untreated PD patients. In addition, dopamine agonists cause nausea and constipation, as do anticholinergics.

Medication for non-motor symptoms may also affect autonomic function. $\alpha$-Adrenergic blockers, which may be used for urinary voiding problems, and antidepressants, also can aggravate orthostatic hypotension and constipation in PD patients.

\section{Effect of deep brain stimulation on the autonomic nervous system}

Deep brain stimulation (DBS) of the subthalamic nucleus (STN) or the globus pallidus internus has become an established tool in the management of patients with advanced PD, and DBS of the STN allows reduction of the daily dose of antiparkinsonian drugs. DBS of the STN may improve autonomic dysfunction, such as bladder problems, ${ }^{74}$ gastric dysmotility ${ }^{42}$ and reduced emotional sweating. ${ }^{75}$ The improvement in autonomic dysfunction may be related to reduced antiparkinsonian drugs, improvement of motor disability and direct effects of DBS on STN and its neighbouring or connecting areas.

\section{AUTONOMIC DYSFUNCTION IN OTHER PARKINSONIAN DISORDERS}

\section{Multiple system atrophy}

Multiple system atrophy (MSA) is characterised by parkinsonism, cerebellar ataxia and autonomic failure. Cardiovascular dysfunction is usually more severe in MSA compared with PD, but some PD patients may present with severe orthostatic hypotension even in the early stages, similar to MSA patients. The presence of orthostatic hypotension alone does not distinguish MSA from PD. ${ }^{52}$ Bladder symptoms, severe voiding symptoms and an excess of $100 \mathrm{ml}$ of residual urine frequently precede postural symptoms in MSA, and they may also precede the development of motor symptoms. ${ }^{76}$ The incidence of constipation is less in MSA compared with PD. ${ }^{77}$

With regard to autonomic tests, most MSA patients show normal myocardial MIBG accumulation, which is useful to differentiate MSA from PD. Previous studies using myocardial MIBG scintigraphy to distinguish PD patients from MSA patients showed sensitivity of $89 \%$ and specificity of $77 \% .^{34}$ The clonidine growth hormone stimulation test may also be useful for differentiating MSA from PD. Clonidine, a selective $\alpha_{2}$-adrenoceptor agonist, evokes release of growth hormone in healthy subjects and PD patients whereas the release is impaired in MSA. ${ }^{78}$ In sphincter electromyography, MSA patients show neurogenic changes in the sphincter motor unit while PD patients show normal activity. ${ }^{49}$ A recent study reported different patterns of EGG activity between MSA and PD. MSA patients showed regular slow wave rhythms with reduced variability, unlike PD patients, who have irregular slow waves. ${ }^{44} \mathrm{~A}$ combination of thermoregulatory sweat testing and the Quantitative Sudomotor Axon Reflex Test were used to differentiate between patients with MSA and $\mathrm{PD}^{79}$; the results showed that the percentage of anhidrosis was significantly larger in MSA than PD and the distribution of anhidrosis was compatible with preganglionic (central) involvement in MSA in contrast with PD which had the peripheral involvement of anhidrosis. Moreover, normal axon reflex sweating in anhidrotic areas confirmed a preganglionic lesion in MSA, although some MSA patients may show reduced axon reflex sweating. ${ }^{80}$ A combination of sympathetic sweat response (SSwR) and skin vasomotor reflex may also be helpful to diagnose MSA, which often shows diminished SSwR and preserved skin vasomotor reflex. ${ }^{81}$

\section{Progressive supranuclear palsy}

Progressive supranuclear palsy (PSP), which is characterised by vertical gaze palsy, dystonia, akinesia, pseudobulbar palsy and mental impairment, is a disorder which has overlapping neurological symptoms and signs with PD. Unlike PD and MSA patients, there are generally no autonomic abnormalities in PSP patients, and cardiovascular autonomic dysfunction should be an exclusion criterion for PSP. $^{82}$ However, pressor responses induced by mental stress ${ }^{82}$ and emotional sweating ${ }^{83}$ have been reported to be diminished in PSP. These findings may reflect cognitive impairment in PSP. MIBG scintigraphy has been reported to show sensitivity of $87 \%$ and specificity of $77 \%$ in the differential diagnosis between PD and PSP. ${ }^{34}$

\section{Dementia with Lewy bodies}

Dementia with Lewy bodies (DLB) is a neurodegenerative disorder characterised by dementia with parkinsonism. Autonomic dysfunction is not uncommon among DLB patients and has 
previously been described in case reports as a preceding feature before dementia or parkinsonism. ${ }^{84}$ An earlier pathological study showed that the majority of confirmed DLB patients reported urinary incontinence and constipation as the most frequent symptoms while almost one-third of these patients experienced episodic hypotension associated with syncopal episodes. ${ }^{85}$ These autonomic features, including repeated falls and syncope, transient loss of consciousness and severe autonomic dysfunction were included in the supportive features in the latest revised criteria for the clinical diagnosis of DLB. ${ }^{86}$ Recently, autonomic function in DLB was systemically evaluated by comparing the characteristics and autonomic symptoms on the Composite Autonomic Scoring Scale and autonomic function tests, including thermoregulatory sweat testing. ${ }^{12}$ The results showed that orthostatic hypotension and Composite Autonomic Scoring Scale scores were abnormal in DLB patients and intermediate between those with MSA and PD. Reduced myocardial MIBG uptake, decreased baroreceptor reflex, ${ }^{87}$ orthostatic hypotension and reduced $\mathrm{SSwR}^{31}$ were more severe in DLB compared with PD. Reduced myocardial MIBG accumulation is also considered as a supportive feature in the diagnostic criteria of DLB. ${ }^{86}$ Myocardial MIBG scintigraphy is also useful to differentiate DLB from other movement disorders, such as $\mathrm{PSP}^{34}$ but MIBG appears to be much more reliable for aiding diagnosis, particularly between DLB and Alzheimer's disease in dementia patients. With regards to bladder dysfunction, recent evidence suggests that DLB may have large post void residuals and neurogenic changes in sphincter motor unit potentials, similar to MSA. ${ }^{88}$

\section{CONCLUSION}

Autonomic features in PD have received increasingly much more attention from clinicians over the past decade, partly due to better knowledge of non-motor symptoms. Previous reports have demonstrated that autonomic dysfunction can present even in the early stages of PD, and some autonomic features sometimes predate the classical motor symptoms. These findings raise the possibility of using them as potential markers for aiding PD diagnosis at an earlier stage.

Contributors MA performed the literature review, and coordinated the conception, authors' writing, revisions and editing, wrote the first draft and finalised the manuscript. EV, DAL and CJM contributed to the literature review, writing of the paper and revisions. VI contributed to revision of the manuscript.

\section{Competing interests None.}

Provenance and peer review Commissioned; externally peer reviewed.

\section{REFERENCES}

1 Abbott RD, Petrovitch $H$, White $L R$, et al. Frequency of bowel movements and the future risk of Parkinson's disease. Neurology 2001;57:456-62.

2 Hawkes $\mathrm{CH}$, Del Tredici K, Braak H. A timeline for Parkinson's disease. Parkinsonism Relat Disord 2010;16:79-84.

3 Lahrmann $\mathrm{H}$, Cortelli P, Hilz M, et al. EFNS guidelines on the diagnosis and management of orthostatic hypotension. Eur J Neurol 2006;13:930-6.

4 Freeman R, Wieling W, Axelrod FB, et al. Consensus statement on the definition of orthostatic hypotension, neurally mediated syncope and the postural tachycardia syndrome. Auton Neurosci 2011;161:46-8.

5 Verbaan D, Marinus J, Visser $M$, et al. Patient-reported autonomic symptoms in Parkinson disease. Neurology 2007;69:333-41.

6 Martinez-Martin P, Schapira AH, Stocchi F, et al. Prevalence of nonmotor symptoms in Parkinson's disease in an international setting; study using nonmotor symptoms questionnaire in 545 patients. Mov Disord 2007;22:1623-9.

7 Goldstein DS, Pechnik S, Holmes C, et al. Association between supine hypertension and orthostatic hypotension in autonomic failure. Hypertension 2003;42: 136-42.

8 Vagaonescu TD, Saadia D, Tuhrim S, et al. Hypertensive cardiovascular damage in patients with primary autonomic failure. Lancet 2000;355:725-6.
9 Garland EM, Gamboa A, Okamoto L, et al. Renal impairment of pure autonomic failure. Hypertension 2009;54:1057-61.

10 Shannon JR, Jordan J, Diedrich A, et al. Sympathetically mediated hypertension in autonomic failure. Circulation 2000;101:2710-15.

11 Jansen RW, Lipsitz LA. Postprandial hypotension: epidemiology, pathophysiology, and clinical management. Ann Intern Med 1995;122:286-95.

12 Thomaides T, Bleasdale-Barr K, Chaudhuri KR, et al. Cardiovascular and hormonal responses to liquid food challenge in idiopathic Parkinson's disease, multiple system atrophy, and pure autonomic failure. Neurology 1993;43:900-4.

13 Micieli G, Martignoni E, Cavallini A, et al. Postprandial and orthostatic hypotension in Parkinson's disease. Neurology 1987;37:386-93.

14 Smith GD, Mathias CJ. Postural hypotension enhanced by exercise in patients with chronic autonomic failure. QJM 1995;88:251-6.

15 Bagheri H, Damase-Michel C, Lapeyre-Mestre $\mathrm{M}$, et al. A study of salivary secretion in Parkinson's disease. Clin Neuropharmacol 1999;22:213-15.

16 Sakakibara R, Uchiyama T, Yamanishi T, et al. Genitourinary dysfunction in Parkinson's disease. Mov Disord 2010;25:2-12.

17 Sakakibara R, Shinotoh H, Uchiyama $T$, et al. Questionnaire-based assessment of pelvic organ dysfunction in Parkinson's disease. Auton Neurosci 2001;92:76-85.

18 Gao X, Chen H, Schwarzschild MA, et al. Erectile function and risk of Parkinson's disease. Am J Epidemiol 2007;166:1446-50.

19 Swinn L, Schrag A, Viswanathan R, et al. Sweating dysfunction in Parkinson's disease. Mov Disord 2003;18:1459-63.

20 Mizuno Y, Takubo H, Mizuta E, et al. Malignant syndrome in Parkinson's disease: concept and review of the literature. Parkinsonism Relat Disord 2003;9(Suppl 1): S3-9.

21 Micieli G, Tassorelli C, Martignoni E, et al. Disordered pupil reactivity in Parkinson's disease. Clin Auton Res 1991;1:55-8.

22 Velseboer DC, de Haan RJ, Wieling W, et al. Prevalence of orthostatic hypotension in Parkinson's disease: a systematic review and meta-analysis. Parkinsonism Relat Disord 2011;17:724-9.

23 Goldstein DS. Cardiovascular autonomic dysfunction. Totowa, NJ: Humana Press, 2005.

24 Puisieux F, Bulckaen H, Fauchais AL, et al. Ambulatory blood pressure monitoring and postprandial hypotension in elderly persons with falls or syncopes. J Gerontol A Biol Sci Med Sci 2000;55:M535-40.

25 Plaschke M, Trenkwalder P, Dahlheim H, et al. Twenty-four-hour blood pressure profile and blood pressure responses to head-up tilt tests in Parkinson's disease and multiple system atrophy. J Hypertens 1998;16:1433-41.

26 DiFrancisco-Donoghue J, Elokda A, Lamberg EM, et al. Norepinephrine and cardiovascular responses to maximal exercise in Parkinson's disease on and off medication. Mov Disord 2009;24:1773-8.

27 Polinsky RJ. Neuropharmacological investigation of autonomic failure. In: Mathias CJ, Bannister R, eds. Autonomic failure a textbook of clinical disorders of the autonomic nervous system, 4th edn. Oxford: Oxford University Press, 2002:232-44.

28 Goldstein DS, Holmes C, Sharabi Y, et al. Plasma levels of catechols and metanephrines in neurogenic orthostatic hypotension. Neurology 2003;60:1327-32.

29 Maetzler W, Liepelt I, Berg D. Progression of Parkinson's disease in the clinical phase: potential markers. Lancet Neurol 2009;8:1158-71.

30 Oka H, Mochio S, Onouchi $\mathrm{K}$, et al. Cardiovascular dysautonomia in de novo Parkinson's disease. J Neurol Sci 2006;241:59-65.

31 Akaogi Y, Asahina M, Yamanaka Y, et al. Sudomotor, skin vasomotor, and cardiovascular reflexes in 3 clinical forms of Lewy body disease. Neurology 2009;73:59-65.

32 Asahina M, Sakakibara R, Liu Z, et al. The raphe magnus/pallidus regulates sweat secretion and skin vasodilation of the cat forepaw pad: a preliminary electrical stimulation study. Neurosci Lett 2007:415:283-7.

33 Goldstein DS, Holmes C, Cannon RO 3rd, et al. Sympathetic cardioneuropathy in dysautonomias. N Engl J Med 1997;336:696-702.

34 Treglia G, Cason E, Stefanelli A, et al. MIBG scintigraphy in differential diagnosis of Parkinsonism: a meta-analysis. Clin Auton Res 2012;22:43-55.

35 Sawada H, Oeda T, Yamamoto K, et al. Diagnostic accuracy of cardiac metaiodobenzylguanidine scintigraphy in Parkinson disease. Eur I Neurol 2009;16:174-82

36 Tipre DN, Goldstein DS. Cardiac and extracardiac sympathetic denervation in Parkinson's disease with orthostatic hypotension and in pure autonomic failure. J Nucl Med 2005;46:1775-81.

37 Matsui $\mathrm{H}$, Udaka F, Oda M, et al. Metaiodobenzylguanidine (MIBG) uptake in Parkinson's disease also decreases at thyroid. Ann Nucl Med 2005;19:225-9.

38 Fuh JL, Lee RC, Wang SJ, et al. Swallowing difficulty in Parkinson's disease. Clin Neurol Neurosurg 1997:99:106-12

39 Bushmann M, Dobmeyer SM, Leeker L, et al. Swallowing abnormalities and their response to treatment in Parkinson's disease. Neurology 1989;39:1309-14.

40 Eadie MJ, Tyrer JH. Radiological abnormalities of the upper part of the alimentary tract in parkinsonism. Australas Ann Med 1965;14:23-7.

41 Bassotti G, Germani U, Pagliaricci S, et al. Esophageal manometric abnormalities in Parkinson's disease. Dysphagia 1998;13:28-31. 
42 Arai $\mathrm{E}$, Arai M, Uchiyama $\mathrm{T}$, et al. Subthalamic deep brain stimulation can improve gastric emptying in Parkinson's disease. Brain 2012;135:1478-85.

43 Chen JZ, MaCallum RW. Electrogastrographic parameters and their clinical significance. In: Chen JD, McCallum RW, eds. Electrogastrography: principles and applications. New York: Raven Press, 1994:45-73.

44 Sakakibara Y, Asahina M, Suzuki A, et al. Gastric myoelectrical differences between Parkinson's disease and multiple system atrophy. Mov Disord 2009;24:1579-86.

45 Sakakibara R, Uchiyama T, Yamanishi T, et al. Bladder and bowel dysfunction in Parkinson's disease. J Neural Transm 2008;115:443-60.

46 Braak H, de Vos RA, Bohl J, et al. Gastric alpha-synuclein immunoreactive inclusions in Meissner's and Auerbach's plexuses in cases staged for Parkinson's disease-related brain pathology. Neurosci Lett 2006:396:67-72.

47 Stocchi F, Badiali D, Vacca L, et al. Anorectal function in multiple system atrophy and Parkinson's disease. Mov Disord 2000;15:71-6.

48 Mathers SE, Kempster PA, Swash M, et al. Constipation and paradoxica puborectalis contraction in anismus and Parkinson's disease: a dystonic phenomenon? J Neurol Neurosurg Psychiatry 1988;51:1503-7.

49 Sakakibara R, Hattori T, Uchiyama T, et al. Videourodynamic and sphincter motor unit potential analyses in Parkinson's disease and multiple system atrophy. J Neurol Neurosurg Psychiatry 2001;71:600-6.

50 Yamamoto T, Sakakibara R, Uchiyama T, et al. Neurological diseases that cause detrusor hyperactivity with impaired contractile function. Neurourol Urodyn 2006;25:356-60.

51 Pavlakis AJ, Siroky MB, Goldstein I, et al. Neurourologic findings in Parkinson's disease. J Urol 1983:129:80-3.

52 Riley DE, Chelimsky TC. Autonomic nervous system testing may not distinguish multiple system atrophy from Parkinson's disease. J Neurol Neurosurg Psychiatry 2003;74:56-60.

53 Kawada M, Tamada Y, Simizu H, et al. Reduction in QSART and vasoactive intestinal polypeptide expression in the skin of Parkinson's disease patients and its relation to dyshidrosis. J Cutan Pathol 2009;36:517-21.

54 Sharabi Y, Li ST, Dendi R, et al. Neurotransmitter specificity of sympathetic denervation in Parkinson's disease. Neurology 2003;60:1036-9.

55 Dabby R, Djaldetti R, Shahmurov M, et al. Skin biopsy for assessment of autonomic denervation in Parkinson's disease. J Neural Transm 2006;113:1169-76.

56 Ikemura M, Saito $Y$, Sengoku R, et al. Lewy body pathology involves cutaneous nerves. J Neuropathol Exp Neurol 2008;67:945-53.

57 Miki Y, Tomiyama M, Ueno T, et al. Clinical availability of skin biopsy in the diagnosis of Parkinson's disease. Neurosci Lett 2010;469:357-9.

58 Gibb WR, Lees AJ. The relevance of the Lewy body to the pathogenesis of idiopathic Parkinson's disease. J Neurol Neurosurg Psychiatry 1988:51:745-52.

59 Wakabayashi K, Takahashi H. The intermediolateral nucleus and Clarke's column in Parkinson's disease. Acta Neuropathol 1997:94:287-9.

60 Orimo S, Takahashi A, Uchihara T, et al. Degeneration of cardiac sympathetic nerve begins in the early disease process of Parkinson's disease. Brain Pathol 2007;17:24-30.

61 Benarroch EE, Schmeichel AM, Low PA, et al. Involvement of medullary regions controlling sympathetic output in Lewy body disease. Brain 2005;128:338-44.

62 Araki I, Kitahara M, Oida T, et al. Voiding dysfunction and Parkinson's disease: urodynamic abnormalities and urinary symptoms. J Urol 2000;164:1640-3.

63 Kujawa K, Leurgans S, Raman R, et al. Acute orthostatic hypotension when starting dopamine agonists in Parkinson's disease. Arch Neurol 2000:57:1461-3.

64 Chase TN, Watanabe AM. Methyldopahydrazine as an adjunct to L-dopa therapy in parkinsonism. Neurology 1972;22:384-92.

65 Worth D, Harvey J, Brown J, et al. The effects of intravenous L-dopa on plasma renin activity, renal function, and blood pressure in man. Eur J Clin Pharmacol 1988;35:137-41

66 Iwasaki S, Hamaguchi K, Iwasaki A, et al. Hypotensive effect of long-term levodopa in patients with Parkinson's disease. Eur Neurol 1990;30:194-9.
67 Senard JM, Verwaerde P, Rascol O, et al. Effects of acute levodopa administration on blood pressure and heart variability in never treated parkinsonians. Hypertens Res 1995:18(Suppl 1):S175-7.

68 Sachs C, Berglund B, Kaijser L. Autonomic cardiovascular responses in parkinsonism: effect of levodopa with dopa-decarboxylase inhibition. Acta Neurol Scand 1985;71:37-42

69 Calne DB, Brennan J, Spiers AS, et al. Hypotension caused by L-dopa. BMJ 1970;1:474-5.

70 Haapaniemi TH, Kallio MA, Korpelainen JT, et al. Levodopa, bromocriptine and selegiline modify cardiovascular responses in Parkinson's disease. J Neurol 2000;247:868-74.

71 Lyytinen J, Sovijarvi A, Kaakkola S, et al. The effect of catechol-0-methyltransferase inhibition with entacapone on cardiovascular autonomic responses in L-Dopa-treated patients with Parkinson's disease. Clin Neuropharmacol 2001;24:50-7.

72 Dive $A$, Foret $F$, Jamart J, et al. Effect of dopamine on gastrointestinal motility during critical illness. Intensive Care Med 2000;26:901-7.

73 Lanfranchi GA, Bazzocchi G, Fois F, et al. Effect of domperidone and dopamine on colonic motor activity in patients with the irritable bowel syndrome. Eur J Clin Pharmacol 1985;29:307-10.

74 Wolz M, Hauschild J, Fauser M, et al. Immediate effects of deep brain stimulation of the subthalamic nucleus on nonmotor symptoms in Parkinson's disease. Parkinsonism Relat Disord. Published Online First: 7 June 2012

75 Priori A, Cinnante C, Genitrini S, et al. Non-motor effects of deep brain stimulation of the subthalamic nucleus in Parkinson's disease: preliminary physiological results. Neurol Sci 2001;22:85-6.

76 Sakakibara R, Hattori T, Tojo $M$, et al. Micturitional disturbance in multiple system atrophy. Jpn J Psychiatry Neurol 1993;47:591-8.

77 Yamamoto T, Sakakibara R, Uchiyama T, et al. Pelvic organ dysfunction is more prevalent and severe in MSA-P compared to Parkinson's disease. Neurourol Urodyn 2011;30:102-7

78 Kimber JR, Watson L, Mathias CJ. Distinction of idiopathic Parkinson's disease from multiple-system atrophy by stimulation of growth-hormone release with clonidine. Lancet 1997:349:1877-81.

79 Lipp A, Sandroni P, Ahlskog JE, et al. Prospective differentiation of multiple system atrophy from Parkinson disease, with and without autonomic failure. Arch Neurol 2009;66:742-50.

80 Iodice V, Lipp A, Ahlskog JE, et al. Autopsy confirmed multiple system atrophy cases: Mayo experience and role of autonomic function tests. J Neurol Neurosurg Psychiatry 2012;83:453-9.

81 Asahina M, Kikkawa $Y$, Suzuki A, et al. Cutaneous sympathetic function in patients with multiple system atrophy. Clin Auton Res 2003;13:91-5.

82 Kimber J, Mathias CJ, Lees AJ, et al. Physiological, pharmacological and neurohormonal assessment of autonomic function in progressive supranuclear palsy. Brain 2000;123:1422-30.

83 Kikkawa Y, Asahina M, Suzuki A, et al. Cutaneous sympathetic function and cardiovascular function in patients with progressive supranuclear palsy and Parkinson's disease. Parkinsonism Relat Disord 2003:10:101-6.

84 Larner AJ, Mathias CJ, Rossor MN. Autonomic failure preceding dementia with Lewy bodies. J Neurol 2000;247:229-31.

85 Horimoto $\mathrm{Y}$, Matsumoto $\mathrm{M}$, Akatsu $\mathrm{H}$, et al. Autonomic dysfunctions in dementia with Lewy bodies. J Neurol 2003;250:530-3.

86 McKeith IG, Dickson DW, Lowe J, et al. Diagnosis and management of dementia with Lewy bodies: third report of the DLB Consortium. Neurology 2005; 65:1863-72.

87 Oka H, Yoshioka M, Morita M, et al. Reduced cardiac 123I-MIBG uptake reflects cardiac sympathetic dysfunction in Lewy body disease. Neurology 2007;69:1460-5.

88 Sakakibara R, Ito T, Uchiyama T, et al. Lower urinary tract function in dementia of Lewy body type. J Neurol Neurosurg Psychiatry 2005;76:729-32. 